\section{Sinus Bone Graft - Where and Where Not to be? - A Review}

\section{Abstract}

The maxillary sinus augmentation procedure has gaining popularity in recent years. The aim of this review article is to provide an update about various indication, contraindication and treatment aspect of the maxillary sinus and their clinical relevance to the sinus augmentation procedure and subsequent implant placement in the atrophic maxilla.

Keywords: Maxillary sinus; Indication; Contraindications; Sinus graft

Received: August 08, 2016; Accepted: August 24, 2016; Published: August 26, 2016

\section{Introduction}

Implant therapy has become an excellent treatment modality since its inception into the modern era of dentistry. The ability to permanently replace missing teeth with a function and appearance close to that of the natural dentition has been possible due to advancement of implant dentistry. Implants are a conservative and esthetic alternative for treatment of partial edentulism and provide a stable foundation for treatment of complete edentulism. However, dental implants can be a viable treatment option only when there is sufficient quantity and quality of bone at the site of implant placement.

The maxillary posterior quadrant offers special challenges to the successful use of implant prostheses to restore dental function. Dental implant placement in the posterior edentulous maxilla could potentially be compromised by the lack of adequate vertical dimension of alveolar bone [1-3]. This occurs due to the proximity of the maxillary sinus to the alveolar crest as a result of sinus pneumatization, as well as resorption of the alveolar ridge owing to tooth extraction, trauma or pathology. Thus, in turn, prevents placement of implants of adequate length. Furthermore, bone density in the posterior maxilla is often poor, which may also lead to a diminished implant success rate.

Several treatment options have been used in the posterior maxilla to overcome the problem of inadequate bone quantity. The most conservative treatment option would be to place short implants to avoid entering the sinus cavity. Another way of avoiding grafting the maxillary sinus would be to place tilted implants in a position mesial or distal to the sinus cavity if these areas have adequate bone. Furthermore, extra-long zygomatic implants can be placed in the lateral part of the zygomatic bone. Of all these techniques, grafting the floor of the maxillary sinus has emerged as the most common surgical modality for correcting

\section{Pranav S Patil, Bhongade ML and Kaustubh S Thakare}

\author{
Sharad Pawar Dental College and Hospital, \\ Wardha, Maharastra, India
}

Corresponding author: Pranav S Patil

$\equiv$ pranavpatil87@gmail.com

Department of Periodontology and Implantology, Sharad Pawar Dental College and Hospital, Sawangi (M), Wardha, Maharashtra-442 005, India.

Tel: 9420349157

Citation: Patil PS, Bhongade ML, Thakare KS. Sinus Bone Graft - Where and Where Not to be? - A Review. Periodon Prosthodon. 2016, 2:3.

this inadequacy. The procedure has been referred to in literature as maxillary sinus augmentation, maxillary sinus lift, subantral augmentation or maxillary sinus floor elevation.

Sinus floor elevation in the atrophic maxillary posterior region to make implantation possible has been increasingly popular in recent years. Two approaches have been described in literature for sinus floor augmentation: the direct approach/lateral approach/external sinus augmentation or the indirect approach/ crestal approach/internal sinus augmentation, using either a one-stage or -two-stage protocol $[4,5]$.

Although maxillary sinus augmentation and implant procedures are compatible, with most patients recovering uneventfully, various intra-operative and postoperative complications have been reported in the literature. These complications are fairly common with both lateral and crestal approach. Therefore, clinician should know detail knowledge regarding the indication and contraindication of maxillary sinus augmentation procedures and also where to go and where not to go for sinus augmentation.

\section{Sinus grafting indications}

Local conditions of the edentulous alveolar ridge, such as loss of alveolar bone height as a result of periodontal disease prior to tooth loss, can make implant placement unfavorable. Distal furcation of the maxillary molar frequently leads to bone loss 
because of facial or palatal inaccessibility for hygiene purposes. Because it is narrow, it is also difficult to curettage the furci to eliminate calculus. Consequently, periodontal disease progresses, and the resulting loss of bone height leads to tooth loss.

\section{Pneumatization}

The maxillary sinus maintains its overall size while the teeth remain in function, but it expands when posterior teeth are lost [6]. The antrum expands both inferiorly and laterally, potentially invading the canine region and even the lateral piriform. After the loss of teeth, the amount of available bone in the posterior maxilla is greatly reduced. This phenomenon is likely the result of atrophy caused by reduced bone strain from occlusal function. Implants placed beneath the ungrafted sinus floor are known to stimulate increased bone formation at the sinus floor. A major criterion for successful implant treatment is availability of bone. A limited review of the literature reveals that implants $10 \mathrm{~mm}$ or less in height have a $16 \%$ lower survival rate than implants greater than $10 \mathrm{~mm}$ in height [7]. Therefore, height of bone is a consideration for predictable implant therapy. Because of periodontal disease, tooth loss, and sinus expansion, there is often less than $10 \mathrm{~mm}$ of bone between the alveolar ridge crest and the floor of the maxillary sinus. In these patients, a phenomenon known as pneumatic trifurcation is often observed, whereby the sinus dips down between the roots nearly to the furcation in the first molar area. Removal of the tooth leaves 4 to $5 \mathrm{~mm}$ of bone as a result of this anatomic sinus peculiarity. Limited vertical dimension compounds the problem of the medialized ridge position and compromised alveolar width. As a result, long-term prognosis is guarded unless these findings are addressed.

\section{Poor bone density}

As a general rule, the quality of bone in the posterior maxilla is poorer than in any other intraoral region [8]. The bone density of the maxilla is often 5 to 10 times lower than that of the anterior mandible [9]. Bone mineral density directly influences the amount of contact between the implant and the bone surface, which transmits load to bone [10]. Bone- implant contact is lowest in D4 bone. Strain pattern spreads farther toward the apex of the implant in poor- density bone than in dense bone [11]. When strain is excessive, bone loss in trabecular bone occurs along the entire implant body instead of only crestally, as occurs in dense bone. Type 4 (D4) bone also has the greatest biomechanical elastic modulus disparity to that of titanium under load. Bone mineral density is critically important for implant survival under load [11]. Implants are at greatest risk of failure under conditions of poor mineralization. A literature review of clinical studies published from 1981 to 2001 reveals that when implants are placed in the poorest bone mineral density, survival is reduced by an average of $16 \%, 5$ with some reports as low as $40 \%$. Deficient osseous structure jeopardizes not only initial implant stability but also load-bearing capacity. Absence of cortex on the ridge crest compromises implant stability, and since the labial cortical plate is usually quite thin and the ridge is relatively wide, it does little to improve stability. Without lateral cortical bone contact to stabilize the implant, initial stabilization in type 4 bone is often compromised.

\section{Strong occlusal forces}

Occlusal forces in the posterior region are greater than in the anterior region of the mouth by as much as a factor of five [12]. Maximum bite force in the anterior region ranges from 241 to $345 \mathrm{~Pa}$. The bite force in the molar region of a dentate individual ranges from 1,378 to 1,723 $\mathrm{Pa}$, [13] Natural maxillary molars have $200 \%$ more surface area as well as a significantly wider diameter than premolars, and both of these factors reduce bone strain. Following the natural tooth model, implant support should be greater in the posterior molar region than in any other area of the mouth. In addition, the posterior maxilla frequently opposes natural teeth or implant-supported restorations, contributing greater force to soft tissue-borne restorations. Therefore, decreased bone quantity and quality as well as increased bite forces should be considered in the treatment of this region of the mouth.

\section{Implant Treatment Planning for the Posterior Maxilla}

\section{Implant size}

The maxillary posterior teeth have the largest diameter, the greatest number of roots, and the largest root surface area of any of the natural dentition. All of these biomechanical strategies help the body to conserve energy (ie, reduce bone strain, sustain greater force to lower-density bone, etc). Implant treatment planning should simulate the function of the natural dentition. Because strain occurs primarily at the crest, implants are designed to minimize unfavorable biomechanical load [14]. Using widediameter implants is an effective means to disperse surface load at the crestal region. While implants of at least 4.0- $\mathrm{mm}$ diameter are generally recommended, a 5.0- $\mathrm{mm}$ implant provides a loadbearing advantage in the molar region. The length of implants used in sinus grafts is generally an empirical choice [15]. In general, threaded root form implants should be at least $12 \mathrm{~mm}$ in length when placed in bone of poor density, as in grafted bone. Implants of this size usually provide adequate bone- implant contact to dissipate the loads applied to the prosthesis (Table 1). Rangert has shown that most of the load occurs at the crestal threads of an implant and that length is not as critical as crestal osseointegration. Therefore, surgeons must determine the crestal bone-healing capacity of a bone graft-directed implant in advance. For this reason, implants of less than $10 \mathrm{~mm}$ in length are generally not advocated.

\section{Number of implants}

Increasing the number of implants is an excellent means of decreasing strain to trabecular bone [16]. Until a more precise method of appraising implant load bearing is devised, one implant for each missing tooth is recommended to support a fixed prosthesis in the posterior maxilla. To further reduce bone strain, implants are generally splinted together. If strain is magnified by para function in the molar region and implant diameter cannot be increased, placing two implants for each missing molar is suggested. 
Table 1 Misch Classification of the Posterior Maxilla.

\begin{tabular}{|c|c|c|c|}
\hline Treatment option & Residual bone Height $(\mathbf{m m})$ & Treatment procedure & Healing time (Months) \\
\hline 1 & $>12$ & Implant placement & Osseointegration 4-6 \\
\hline 2 & $10-12$ & Sinus graft: simultaneous implant placement & $\begin{array}{c}\text { Graft consolidation 2-4 } \\
\text { Osseo integration 4-8 }\end{array}$ \\
\hline 3 & $5-10$ & Lateral wall approach sinus graft: delayed & implant placement \\
\hline 4 & $>5$ & Lateral wall approach sinus graft: delayed & Graft consolidation 6-10 \\
Osseo integration 4-10
\end{tabular}

\section{Implant design}

Surface roughness increases the surface area for osseointegration. A threaded implant has $30 \%$ to $200 \%$ more surface area than a cylindrical implant. Although threaded implants are more difficult to place, their use in low- density bone is strongly recommended. Biomechanical features of thread design (i.e., thread pitch, shape, and depth) further influence the amount of surface area available.

\section{Contraindications for Implant Treatment in the Posterior Maxilla}

A key to long-term success of implant therapy in the posterior maxilla is the presence of an adequate number of anterior teeth or implants. It is a rule in traditional prosthetics that if the canine and two adjacent teeth are missing, a fixed prosthesis is contraindicated [17]. This is because the length of the span results in 27 times the metal flexure resulting from a single pontic span. The amount and direction of the force at the canine region increases to such an extent that a fixed prosthesis should not be considered, regardless of the number of teeth that are splinted together. Therefore, when (a) both premolars, or (b) the first premolar and the lateral incisor, or (c) the lateral and central incisors are missing, along with the canine, a fixed restoration is contraindicated. A removable prosthesis that has no movement under function is considered a fixed prosthesis in terms of the number and position of the implants. Therefore, the treatment plan should provide for maintenance or restoration of healthy anterior teeth or placement of root form implants. Before posterior implants are considered, a healthy natural tooth or implant abutment is required in the canine region of the quadrant. Crown height space should also be evaluated prior to implant placement. When the occlusal plane has been properly positioned, the crown height space should be greater than $8 \mathrm{~mm}$ [18]. If less than $8 \mathrm{~mm}$ is available, excess tissue can be removed via gingivoplasty; otherwise, osteoplasty or vertical impaction osteotomy of the alveolar process is indicated to restore interocclusal space. Abnormal intraoral conditionscan compromise the outcome of sinus grafting procedures and jeopardize the survival of the implants. Dental contraindications to sinus grafting include inadequate oral hygiene, untreated periodontal disease of the residual dentition, severe malocclusion, and severe clenching or bruxism.

\section{Conventional implant placement}

The first treatment option requires the presence of sufficient bone height to permit placement of endosteal implants following standard surgical protocol.

\section{Sinus augmentation}

The second option requires 10 to $12 \mathrm{~mm}$ of vertical bone. Vertical bone for improved implant survival (division A) is developed via an osteotome technique through the alveolar crest $[19,20]$. The osteotomy is performed approximately 1 to $2 \mathrm{~mm}$ below the floor of the sinus using a flat-end osteotome firmly tapped 2 to $3 \mathrm{~mm}$ beyond the prepared implant osteotomy. A greenstick up fracture of the sinus floor elevates the bone and sinus membrane above the blunt end of the osteotome. A longer implant is then placed into the osteotomy, extending into the sinus cavity 2 to 3 $\mathrm{mm}$ beyond the available bone. It is not necessary to place bone graft material with so slight an elevation. The success of sinus bone augmentation that is accomplished through the implant site cannot be confirmed at the time of implant placement. Four to six months later, radiographic evidence of bone growth at the sinus floor demonstrates successful augmentation. Sinus perforation during the osteotome technique or implant placement will prevent bone formation from occurring. However, even if no bone forms around the apical portion of the implant, the cortical-like bone lining of the sinus engages the implant in the apical third region and improves implant rigidity and stress transfer to the bone-implant interface. When alveolar width is insufficient (i.e., division B or division C), onlay bone grafts are used to gain width either at the time of sinus elevation or after a healing delay.

\section{Sinus graft and immediate or delayed implant placement}

When a minimum of $5 \mathrm{~mm}$ of vertical bone is present between the antral floor and the crest, a third approach to the maxillary posterior edentulous region is indicated. A lateral approach to sinus augmentation is combined with onlay grafting when the original ridge width is narrow. If the ridge is adequate, the implant is placed at the same time as the sinus augmentation. When implant stability is questionable, a delayed approach is recommended. A review of the literature on sinus grafts and simultaneous implant placement revealed success rates ranging from $75 \%$ to $97 \%$ [21]. Implants are lost most often because of postoperative complications such as infections. The infection rate is approximately $3 \%$ higher when implants are placed in conjunction with a sinus graft than when implants are placed in native bone [22]. When infection occurs, a bacterial smear layer forms over the implant surface, dramatically compromising the conditions necessary for osseointegration. The presence of an implant within the bone graft makes treatment of the infection more difficult. It is prudent to delay implant placement until the graft has consolidated in patients who are susceptible to infection, such as smokers and patients with poorly controlled diabetes. In 
addition, delayed implant placement into a healed graft allows the surgeon to assess bone quantity and quality at the same time. The delayed approach fixates the implant more rigidly than is possible during immediate placement because the apical portion of the implant engages mature bone graft. The surgeon also is able to assess the availability of vertical bone when a delayed approach is used, avoiding inadvertent antral placement of the implants in "underaugmented" or resorbed sinus grafts. Upon reentry to a sinus graft, it is not unusual to observe a craterlike bone defect at the lateral access window with attendant soft tissue invagination. This finding may relate to how densely the graft was placed or to the use of a barrier membrane. If the implant is already in place in a poorly consolidated sinus graft, it may be difficult to remove nonossified tissues and accurately assess osseointegration. If possible, soft tissue is curetted and the site is regrafted. Consideration should be given to removing an implant that is only partially integrated. The healing time for an implant placed into grafted bone depends on the type of graft materials used as well as host healing capacity. Bone graft healing is related in part to the transantral dimension (medial to lateral wall). If this dimension is small ( 0 to $5 \mathrm{~mm}$ ), healing time is optimal; if medium ( 5 to $15 \mathrm{~mm}$ ), healing time is prolonged; if large (greater than $15 \mathrm{~mm}$ ), healing time may be extended since graft material is further away from the endosteal blood supply. The suggested healing time after implant placement for these morphologies is 4,6 and 8 months, respectively. In addition, a wide-flat sinus morphology suggests the use of autologous bone as the primary sinus graft material.

\section{Delayed implant placement}

When alveolar ridge height is $4 \mathrm{~mm}$ or less, a delayed approach is advocated. At about 3 to $4 \mathrm{~mm}$, the host bone is insufficient to provide implant stability at the time of sinus augmentation, and implant placement should be delayed. This option depends on minimal host bone, thus the need for greater graft volume. A compromised osseous bed, extensive pneumatization, and insufficient bone structure for primary implant stabilization require more time for bone graft consolidation prior to implant placement unless morphogenetic cell-based therapies are uses. Regardless of the healing time before re-entry, the surgeon must consider sinus morphology, sinus depth (small, medium, or large), the amount of autologous bone in the graft, and overall graft volume when assessing the chances for success. The design of the implant, as in any region, depends on the amount and the morphology of available bone and the prosthesis that is planned. In a staged approach, the bone graft is evaluated again at implant placement and after at least 4 additional months have elapsed for bone graft remodeling before prosthodontic reconstruction.

Sinus grafting has been demonstrated to be a safe and predictable procedure for the correction of the atrophic edentulous maxilla, whether it is accomplished alone or in conjunction with other reconstructive procedures, such as bone grafts, guided bone regeneration, or distraction osteogenesis. Disuse remodeling of the sinus and posterior maxillary morphology following tooth loss suggests various treatment options based on site classification. When approached and managed properly, these techniques lead not only to implant survival but also to restoration of interarch orthoalveolar form and function.

\section{Contraindications for Sinus Graft Procedures}

As with any surgical procedure, a number of potential complications of sinus floor augmentation may interfere with the normal function of the sinus. It is important to understand maxillary sinus function and how it is affected by the sinus graft procedure. There are limits to this treatment modality. Medical and surgical risk factors should always be addressed before a decision is made to proceed with surgery. Risks and benefits are important and should be considered and discussed with the patient.

Proper function of the maxillary sinus depends on a delicate balance between mucus production, transport by ciliated epithelium, sinus ventilation, and sustainable drainage through the ostium. In addition, there are communicating ethmoid and frontal sinuses that affect the maxillary sinus if they are unhealthy or chronically inflamed. These conditions can arise unilaterally or bilaterally. Any factor interfering with one of these functions will compromise maxillary sinus health. A grafting procedure generally does not interfere with sinus function when performed on a healthy sinus [23-28]; however, when performed on an unhealthy sinus, the same procedure will contribute to fluid stagnation and bacterial overgrowth, leading to an exacerbated sinusitis. Moreover, the presence of space-occupying masses such as polyps, tumors, and hyperplastic mucosa represent obstacles to the elevation of the sinus mucosa. Pre-existing local pathologic conditions represent relative or absolute contraindications to the sinus graft procedure and therefore must be carefully scrutinized before surgery. Sinus-grafting procedures are performed to enable implant placement and rehabilitation with implantsupported prostheses. Therefore, all intraoral contraindications for the placement of dental implants must be considered as well.

Finally, as with all surgical procedures, the systemic medical health of the patient must be evaluated. All patients who undergo sinus surgical procedures should receive a thorough medical evaluation. The degree and type of surgery, type of anesthesia, and general health of the patient are all critical factors that must be reviewed to establish candidacy.

\section{Local Contraindications}

Local contraindications fall into two main groups: (1) potentially reversible (relative); and (2) irreversible (absolute). The first group includes pathologies that, if not treated, contraindicate sinus grafting. The second group includes pathologies that, even after surgical management, leave irreversible dysfunction of the osteometal complex.

\section{Potentially reversible, relative contraindications to sinus grafting}

Some anatomic and/or structural alterations of the nasomaxillary complex may interfere with normal ventilation and mucociliary clearance of the maxillary sinus. Compensation may occur over time, leaving such abnormal conditions clinically silent or with only mild to moderate, sometimes intermittent, symptoms. Sinusgrafting procedures in this setting decompensate a compromised 
sinus, causing mucus stasis, supra infection, and subacute sinusitis. The elevation of the sinus floor and/or modification of sinus anatomy may on occasion lead to better sinus drainage in the presence of mild sinus membrane dysfunction. But in general, alterations in function of the maxillary sinus membrane and the osteomeatal complex should be identified and treated before sinus grafting is performed. It is imperative that patients undergo thorough radiographic evaluation to identify underlying sinus pathology and anatomic disturbance. Computerized tomography (CT) scans, plain film radiographs, and patient history are part of thecomprehensive workup of the patient.

Positive radiographic findings include:

1.Narrowing of the osteomeatal complex due to a deviated septum; abnormal morphology of the middle turbinate; enlargement of air cells within the middle turbinate, known as concha bullosa; enlargement of an air cell in the roof of the sinus (Haller cell); medial or lateral rotation of the uncinate process; enlargement of the bulla ethmoidale with narrowing of the uncinate process; or post-traumatic or postsurgical scarring.

2.Benign tumors of the nasomaxillary complex such as papillomas, schwannomas, osteomas, polyps, or mucus retention cysts.

3.Viral, bacterial, and micotic rhinosinusitis.

4.Malignancy of the nasomaxillary region.

Treatment of the offending etiology (eg, endodontic treatment of necrotic teeth, medical or surgical therapy of sinusitis, removal of tumors or polyps) must eradicate the pathologic condition prior to sinus graft augmentation. Bacterial sinusitis of the left maxillary sinus and mucus retention cyst on the right sinus. Micotic sinusitis caused by intrasinusal foreign body. Resolution of a compromised sinus is accomplished by functional endoscopic sinus surgery. This approach corrects anatomic or structural alterations of the nasomaxillary complex and removes pathologic sinus tissue that is otherwise not susceptible to medical management [29]. After functional endoscopic sinus surgery, if radiographic and/or endoscopic evidence demonstrates resolution of the pre-existing pathology, it is possible to perform a sinus graft procedure.

\section{Irreversible, absolute contraindications to sinus grafting}

Some anatomic and/or structural alterations or pathologies of the nasomaxillary complex may represent absolute contraindications to the sinus graft procedure. These include:

1. Severe (non correctable) deformities of the maxillary sinus

2. Scarred and hypofunctional sinus mucosa following trauma or previous operation

3. Radiotherapy of the head and neck area (radiation dose above 45 Gy60)

4. Chronic recurrent sinusitis, with or without polyposis, that disrupts mucociliary clearance and is unresponsive to medical or surgical treatment
5. Local expression of a systemic granulomatous disease such as Wegener granulomatosis or midline idiopathic granuloma

\section{Sarcoidosis}

7. Benign but locally aggressive tumor (eg, ameloblastoma, myxoma, desmoplastic fibroma, inverted papilloma)

8. Malignant tumor, both primary and metastatic, deriving from epithelial, connective, or odontogenic tissues (eg, squamous cell carcinoma, esthesioneuroblastoma, adenoid cystic carcinoma, adenocarcinoma, sarcoma). These tumors may require extensive resection and may permanently disturb mucociliary function.

\section{Intraoral Contraindications}

Abnormal intraoral conditions may compromise the sinusgrafting procedure and/or survival of dental implants placed into the grafted sinuses. These contraindications are similar to those reported in non-sinus-directed implant locations and include the following:

1. Grossly inadequate oral hygiene or inability to perform or maintain appropriate oral hygiene

2. Untreated periodontal disease of adjacent dentition

3. Gross malocclusion and insufficient freeway space for restoration

4. Severe pathologic parafunctional habit (clenching or bruxism)

5. Fulminant mucosal disease (desquamative mucosal disease, erosive lichen planus)

6. Severe xerostomia

\section{General Medical Conditions to be Determine}

Compromised general health may represent a relative or absolute contraindication to sinus grafting. Generally speaking, systemic pathoses, such as increased risk for myocardial infarction, hypertensive crisis, or sudden hypoglycemia, may proscribe surgical intervention. The sinus graft procedure should be avoided in patients with compromised healing, such as patients with uncontrolled diabetes, immunocompromised patients, or patients on antitumoral chemotherapy.

The following conditions, unless treated and under control with the patient's complete understanding of the risks, generally contraindicate the sinus graft procedure:

1. Chronic renal disease

2. Chronic liver disease

3. Uncontrolled diabetes

4. Uncontrolled hypertension

5. Hemophilia or treatment with anticoagulant therapy

6. Metabolic bone disorders 
7. Uncontrolled thyroid disorders

8. Uncontrolled adrenal disorders

9. Immunocompromise, including HIV

10. Steroid treatment at the time of the sinus graft procedure

11. Pregnancy

\section{General Surgical Contraindications Include}

1. Chemotherapy for the treatment of malignant tumors at the time of the sinus graft procedure

2. Radiotherapy

3. Drug or alcohol abuse

4. Heavy smoking

5. Physical or psychiatric handicaps

6. Patient noncompliance

\section{Myocardial Infarction}

A history of myocardial infarction, particularly recent infarction (within the previous 6 months), may represent a contraindication. With the exclusion of recent myocardial infarction, after thorough analysis of ventricular function, compromised patients should be treated in a hospital setting. Generally, elective surgery should be delayed 6 months after infarction. Stable medical management and medical clearance for the sinus graft procedure should be obtained. A critical factor of concern in a patient with a history of myocardial infarction is the severity of myocardial ischemia, ventricular irritability, and ventricular ejection fraction. It is imperative that elective dental procedures be delayed 6 months after infarction. Close communication with the patient's treating physician is strongly recommended to avoid inordinate medical risk [30].

\section{Other Cardiac Pathologies}

Patients with cardiac prosthetic valves, a history of subacute bacterial endocarditis, congenital malformation, rheumatic heart disease, sequelae of vascular surgery, cardiomyopathy, or vascular disease with regurgitation may be treated with sinus grafting, but they require antibiotic prophylaxis. Patients with an isolated nongrafted septal defect, mitral valve prolapse without regurgitation, functional murmur, or cardiac pacemaker, and those who have undergone coronary bypass are treated without antibiotic prophylaxis.

\section{Anticoagulant Therapy}

Patients who take anticoagulants may undergo a sinus graft procedure, but only after authorization of the patient's treating physician to verify coagulation status [31,32]. Discontinuing anticoagulants is no longer considered absolutely necessary if the INR value is within an acceptable range. Many patients today self-administer aspirin or holistic vitamin therapy. These can significantly affect bleeding time and coagulation. Discontinuing these medications at least 1 week prior to surgery is appropriate.

\section{Radiotherapy and Chemotherapy}

Definitive data are lacking on the question of whether a prolonged interval is needed between radiation and implant placement or bone-grafting procedures. Some authors [33-38] advocate an extended waiting period between irradiation and rehabilitation surgery in order to avoid complications, recommending at least 12 months prior to implant placement [39-41]. Hyperbaric oxygen therapy may also be helpful in reducing the risk of radionecrosis or failure of bone incorporation [42-47]. In general, irradiated patients who undergo open-flap osseous surgery should be treated with extreme caution.

A conservative approach is to delay implant placement or grafting procedures until 3 months after chemotherapy so that the hematopoietic response can return to within normal limits. The patient must have physician clearance for the procedure and must understand the added risk should the disease recur and additional chemotherapy be required. Patients with bone grafts and implants that are already in place, who then undergo chemotherapy, seem to do well. However, the risk of infection, negative tissue response, and so forth is increased.

Due to the relevant side effects of chemotherapy and the insufficient information concerning its effects on sinus- grafting procedures, extreme caution is suggested when treating these patients. Current chemotherapy (in particular, high-dose chemotherapy) for malignant tumors represents an absolute contraindication for sinus-grafting procedures. Moreover, these patients generally present with active malignancies and an uncertain prognosis, a further contraindication for an elective surgical procedure. Low- dose chemotherapy or a history of previous chemotherapy with an acceptable general health condition may represent only a relative contraindication. In these cases, contacting the treating oncologist and ascertaining the status of the patient's immune system is suggested. The patient's white blood cell count and platelet status must be determined before surgery. In general, surgical procedures may be performed if the granulocyte count is above $2,000 / \mathrm{mm}$ and the platelet count is above $40,000 / \mathrm{mm}$ [48].

\section{Bisphosphonate Therapy}

There has been growing awareness of compromised jawbone healing in patients receiving bisphosphonate therapy. Based on several reports in the literature, it appears that the pathogenesis of this process is most consistent with a defect in jawbone healing and/or localized vascular insufficiency [49-52]. Hypothetically, the mechanism by which bisphosphonates can have this effect may be related to their effect on osteoclasts. With significant impairment of osteoclast function, normal bone turnover and resorption are inhibited. This could result in decreased new bone formation and diminished capillary ingrowth. Bisphosphonates have also demonstrated effects unrelated to osteoclast inhibition. Pamidronate was reported to significantly depress bone blood flow in rats $[53,54]$. The mechanism of this effect may be attributable to a complex interaction of pamidronate with growth hormone and insulin-like growth factor I, both of which are thought to play a role in the regulation of blood circulation in bones. In a recent study, bisphosphonates were shown to inhibit 
endothelial cell function in vitro and in vivo [55]. Cells treated with bisphosphonates demonstrated decreased proliferation, an increased rate of apoptosis, and a decrease in capillary tube formation [56]. Bisphosphonates have also demonstrated antiangiogenic properties due to their ability to significantly decrease circulating levels of vascular endothelial growth factor (VEGF, a potent angiogenic factor) in breast cancer patients with bone metastases [57]. Furthermore, these bisphosphonate properties could explain the apparent ischemic changes noted in the affected patients' jawbones.

Management of patients with bisphosphonate-related osteonecrosis is extremely difficult. Surgical debridement has not been effective in eradicating the necrotic bone, and hyperbaric oxygen therapy has not been uniformly effective in limiting the progression of this process. It is difficult to obtain a surgical margin with viable bleeding bone. Therefore, surgical treatment should be avoided in most cases. Areas of necrotic bone that are a constant source of soft tissue irritation should be removed without exposing additional bone. However, it is likely that the margin of the debridement will remain exposed. Symptomatic patients with pathologic mandibular fractures may require a segmental resection with a continuity defect and immediate reconstruction with a rigid plate. Reconstruction of these patients with free or vascularized bone is not feasible given the likelihood that necrotic bone will be present or develop at the resection margin. Most patients with limited areas of exposed bone can be managed with irrigations and antibiotic therapy that is tailored to the culture and sensitivity data. Cessation of bisphosphonate treatment has not had a major impact on the osteonecrosis.

\section{Smoking}

Smoking is known to be associated with an increased susceptibility to allergy and infections because it interferes with ciliary function and secretory immunity of the nasorespiratory tract. In the maxillary sinus, this may affect immune exclusion and suppression because both surface immunoglobulin $A(s \lg A)$ and slgM responses are reduced, whereas IgE responses are increased. Smoking disturbs bone graft healing because it reduces local blood flow by increasing peripheral resistance and platelet aggregation. By-product chemicals such as hydrogen cyanide and carbon monoxide inhibit wound healing, as does nicotine, which inhibits cellular proliferation. Although smokers may be treated with sinus-grafting procedures, smoking represents a relative contraindication because of the risk of wound dehistence, graft infection and/or resorption, and a $10 \%$ reduced probability of osseointegration.

\section{Osteoporosis}

The concern that patients with osteoporosis are at an increased risk for dental implant failure is based on the assumption that the impaired bone metabolism in other areas of the skeleton affects the mandible or maxilla in a similar manner. However, whether there is a relationship between osteoporosis and decreased oral bone mass or density is controversial [58-60]. There is controversy in the literature, in both clinical and experimental studies, regarding the survival rate of implants placed into osteoporotic bone [61-64]. Both human trials and animal studies indicate that implant therapy is generally successful in osteoporotic bone. No study to date has proven an association between implant failure and osteoporosis. Therefore, the available data do not contraindicate sinus grafting for patients with osteoporosis. Considerations for the osteoporotic patient include:

1. Prior to sinus grafting, the osteoporotic patient should undergo a comprehensive assessment, including endocrinologic, orthopedic, and, if necessary, obstetric examination. A therapeutic regimen of physiologic doses of vitamin D (from 400 to $800 \mathrm{lU} /$ day) and calcium (1,500 $\mathrm{mg} /$ day) is recommended. Calcitonin, which inhibits bone resorption and alters calcium metabolism, may be prescribed. Patients with osteoporosis should continue this regimen throughout the healing period following grafting procedures. Smoking significantly increases the overall risk for demineralization and subsequent failure of osseointegration in patients with osteoporosis.

2. In patients with insufficient alveolar bone, such as highly pneumatized sinuses, implant sites should be augmented prior to implant placement to increase bone support. Simultaneous implant and grafting procedures should be avoided.

3. A clinical evaluation of bone density should be performed at the time of implant placement to reduce the risk of implant instability. Osteoporosis may prevent biomechanical fixation of osseointegrating implants. An implant design that will ensure primary stability in osteoporotic bone of reduced density is preferred. Assessment of the bone quality at the time of the grafting and/or implant procedure may be aided by a careful analysis of radiographs (especially CT scans or radiofrequency analysis), which provide more information about implant failure expectation than peripheral dualenergy x-ray absorptiometry of the radius or ulna.

At present there is no basis for advocating immediate loading of dental implants to enhance osteogenesis in osteoporotic bone [65].

\section{Diabetes}

Diabetes is associated with a wide range of systemic complications such as retinopathy, micro- and macrovascular disease, altered wound healing, and susceptibility to infection. In the oral cavity, diabetes is associated with xerostomia, increased levels of salivary glucose, and an increased incidence of periodontal disease. In particular, the risk of developing periodontitis is significantly higher in patients with diabetes than in nondiabetic patients. This increased susceptibility may be due to a compromised host defense system (exemplified by decreased chemotaxis and phagocytosis, as well as decreased bactericidal action of polymorphonuclear neutrophil leukocytes). In addition, microvascular disease adversely affects the blood supply and contributes to a higher susceptibility to infection [66]. Regarding the impact of diabetes on the healing response to endosseous implants, studies using animal models have shown significantly 
reduced bone-implant contact compared to nondiabetic controls [67]. Although the total bone-implant contact is lower in diabetic than in nondiabetic animals, osseointegration is primarily reduced in trabecular bone, whereas no difference was shown in cortical bone [68]. This finding indicates that although the healing process in animals with uncontrolled diabetes is impaired, osseointegration will occur especially in the mandible. In the maxilla (and hence in bone-grafted sinuses) where trabecular bone predominates, one would expect an increased risk for implant failure. Several studies have specifically addressed the failure rate of dental implants in the diabetic patient, [6972] but little information is available for diabetes and the sinus graft procedure. Definitive guidelines about implant treatment for diabetic patients have not been established, but studies do indicate that diabetes must remain a relative contraindication to implant placement. Although a majority of the published studies are concerned with the influence of diabetes on dental implants rather than on sinus bone grafting, the following guidelines should be considered:

1. Good metabolic control of diabetic patients is recommended. When implants are placed in patients with well-controlled diabetes, successful osseointegration is the same as in the general population [73]. Patients who do not demonstrate strict metabolic control should be metabolically optimized before reconstructive procedures are attempted.

2. There is general agreement in advocating the use of antibiotic prophylaxis in diabetic patients; the use of chlorhexidine mouth rinse is also recommended to reduce oral bacterial count [74-77].

\section{Alcohol Abuse}

Alcohol abuse produces a variety of deleterious systemic effects, including decreased liver function, cardiomyopathies, anemias, and neurologic events. Although no definitive guidelines have been established, the degree of alcohol abuse may suggest either a relative or an absolute contraindication [78]. Patients who abuse alcohol frequently present with poor oral hygiene and noncompliance, which reduces the prospect for a favorable prognosis of sinus-directed implants.

\section{Thyroid Disorders}

Surgical concerns related to a patient with hypothyroidism are a decreased metabolic rate and significant potential for hypotension. The most common causes of hypothyroidism are Hashimoto thyroiditis, idiopathic hypothyroidism, and surgical or radiation treatment to the thyroid. Patients with hyperthyroidism need to be carefully watched for thyroid storm, which is a life-threatening condition. The most common causes of hyperthyroidism are graves disease, toxic nodular goiter, and subacute thyroiditis. Medical clearance and careful medical follow-up are strongly recommended.

\section{Adrenal Disorders}

Although less common than the preceding medical problems, adrenal disorders raise significant concerns for elective treatment. Risks associated with adrenal disorders include shock, dehydration, abdominal pain, nausea, and vomiting. All these events need to be carefully monitored in patients with any history of adrenal disease and in those whose disease is suppressed by steroid therapy. Steroid supplementation is recommended prophylactically; following is the most common regimen:

1. Hydrocortisone sodium succinate, $100 \mathrm{mg}$ intravenously, available in operating room

2. Hydrocortisone, $50 \mathrm{mg}$ intravenously, every 6 hours for the first 24 hours

3. Hydrocortisone, $25 \mathrm{mg}$ intravenously, every 6 hours for 3 to 5 days postoperatively. If the postoperative course is complicated by fever or hypotension, the hydrocortisone dose must be increased.

\section{Summary}

Sinus bone grafting for gaining bone mass in the atrophic edentulous maxilla has been demonstrated to be both safe and predictable. The modifications of sinus morphology by sinus membrane elevation and bone grafting do not jeopardize sinus function unless there is pre-existing sinus pathology. Sinus graft failure is generally avoided by careful preoperative clinical and radiographic examination to optimize the chance for success. Otolaryngologic procedures, such as functional endoscopic surgery, resolve most sinus pathology so that sinus grafts can be accomplished. Overall, the contraindications to sinus grafting must be based on sound clinical judgment with particular emphasis placed on healthy sinus function and systemic vigor. Absolute and relative contraindications for the sinus bone graft procedure take into account systemic manifestations of disease as well as local pathophysiology. The experienced surgeon will confer with medical colleagues and defer treatment until optimal medical management is possible and general operative risk is low. 


\section{References}

1 Bryant SR (1998) The effects of age, jaw site, and bone condition on oral implant outcomes. Int J Prosthodont 11: 470-490.

2 Truhlar RS, Orenstein IH, Morris HF, Ochi S (1997) Distribution of bone quality in patients receiving endosseous dental implants. J Oral Maxillofac Surg 55: 38-45.

3 Adell R, Lekholm U, Rockler B, Brånemark PI (1981) A 15 year study of osseointegrated implants in the treatment of the edentulous jaw. Int J Oral Surg 10: 387-416.

4 Stelzle F, Benner KU (2011) Evaluation of different methods of indirect sinus floor elevation for elevation heights of $10 \mathrm{~mm}$ : An experimental ex vivo study. Clin Implant Dent Relat Res 13: 124-133.

5 Sani E, Veltri M, Cagidiaco MC, Balleri P, Ferrari M (2008) Sinus membrane elevation in combination with placement of blasted implants: A 3-year case report of sinus augmentation without grafting material. Int J Oral Maxillofac Surg 37: 966-969.

6 Misch CE (1993) Treatment planning for edentulous maxillary posterior region. In: Misch CE (ed). Contemporary Implant Dentistry. St Louis: Mosby, pp: 241-255.

7 Goodacre JC, Bernal G, Riringcharassaeng K, Kan JY (2003) Clinical complications with implants and implant prostheses. J Prosthet Dent 2: 121-132.

8 Misch CE (1988) Bone character: Second vital implant criterion. Dent Today 7: 39 .

9 Misch CE, Qu Z, Bidez MW (1999) Mechanical properties of trabecular bone in the human mandible: Implications for dental implant treatment planning and surgical placement. J Oral Maxillofac Surg 57: 700-706.

10 Misch CE (1990) Density of bone: Effect on treatment plans, surgical approach, healing and progressive loading. Int J Oral Implantol 6: 23-31.

11 Misch CE (1993) Bone density. In: Misch CE (ed). Contemporary Implant Dentistry. St Louis: Mosby, USA, pp: 241-255.

12 Scott I, Ash MM (1966) A six-channel intra-oral transmitter for measuring occlusal forces. J Prosthet Dent 16: 56.

13 Anderson DJ (1958) Measurements of stress in mastication. J Dent Res 35: 644-671.

14 Misch CE, Bidez MW (1999) A scientific rationale for dental implant design. In: Misch CE (ed). Contemporary Implant Dentistry, St Louis, Mosby Publishers, USA, pp: 329-343.

15 Misch CE (1999) Divisions of available bone. In: Misch CE (ed). Contem-porary Implant Dentistry, St Louis: Mosby, USA, pp: 89-107.

16 Bidez MW, Misch CE (1992) Force transfer in implant dentistry: Basic concepts and principles. Oral Implantol 18: 264-274.

17 Shillingburg HE, Hobo S, Whitsett LD (1997) Fundamentals of Fixed Prosthodontics. Chicago: Quintessence publishers.

18 Lindquist LW, Carlsson GE, Jemt TA (1996) A prospective 15-year follow-up study of mandibular fixed prostheses supported by osseointegrated implants. Clinical results and marginal bone loss. Clin Oral Implants Res 7: 329-336.

19 Jensen OT, Leonard BS, Block MS, lacono VJ (1998) Report of the Sinus Consensus Conference of 1996. Int J Oral Maxillofac Implants 13: $11-30$.

20 Valentini P, Abensur DJ (2003) Maxillary sinus grafting with anorganic bovine bone: A clinical report of long-term results. Int J Oral Maxillofac Implants 18: 556-560.
21 Stammberger $H$ (1989) History of rhinology: Anatomy of the paranasal sinuses. Rhinology 27: 197-210.

22 Valentini P, Abensur DJ (2003) Maxillary sinus grafting with anorganic bovine bone: A clinical report of long-term results. Int J Oral Maxillofac Implants 18: 556-560.

23 Regev E, Smith RA, Perrot DH, Pogrel MA (1995) Maxillary sinus complications related to endosseous implants. Int J Oral Maxillofac Implants 10: 451-461.

24 Timmenga NM, Raghoebar GM, Boering G, van Weissenbruch R (1997) Maxillary sinus function after sinus lifts for the insertion of dental implants. J Oral Maxillofac Surg 55: 936-939.

25 Zimbler MS, Lebowitz RA, Glickman R (1998) Antral augmentation osseointegration and sinusitis: The otolaryngologist perspective. Am J Rhinol 12: 311-316.

26 Watelet JB, Van Cauwenberge P (1999) Applied anatomy and physiology of the nose and paranasal sinuses. Allergy 54: 14-25.

27 Peleg M, Chaushu G, Mazor Z (1999) Radiological findings of the post-sinusitis lift maxillary sinus: A computerized tomography followup. Periodontol 70: 1564-1573.

28 Van den Berg JP, Ten Bruggenkate CM, Disch FJ, Tuinzing DB (2000) Anatomical aspects of sinus floor elevations. Clin Oral Implants Res 11: $256-265$.

29 Sambataro G, Mantovani M, Scotti A (2003) Rialzo del seno mascellare e implicazioni sulle sue funzioni. In: Chiapasco M, Romeo E (eds). La riabilitazione implanto-protesica nei casi complessi. Torino, Italy, pp: 292-298.

30 Goldman L (1983) Cardiac risks and complications of noncardiac surgery. Ann Intern Med 98: 504-513.

31 Goldman L, Caldera DL, Nussbaum SR (1977) Multifactorial index of cardiac risk in noncardiac surgical procedures. N Engl J Med 297: 845-850.

32 Cygan R, Watzkin H (1987) Stopping and restarting medications in the perioperative period. J Gen Intern Med 2: 270.

33 Marx RE, Johnson RP (1987) Studies in the radiobiology of osteoradionecrosis and their clinical significance. Oral Surg Oral Med Oral Pathol 64: 379-390.

34 Epstein JB, Wong FLW, Stevenson-Moore P (1987) Osteoradionecrosis: Clinical experience and a proposal for classification. J Oral Maxillofac Surg 45: 104-110.

35 Nguyen TD, Panis X, Froissart D, Legros M, Coninx P, et al. (1988) Analysis of late complications after rapid hyperfractionated radiotherapy in advanced head and neck cancers. Int J Radiat Oncol Biol Physics 14: 23-25.

36 Widmark G, Sagne S, Heikel P (1989) Osteoradionecrosis of the jaws. Int J Oral Maxillofac Surg 18: 302-306.

37 Murray CG, Herson J, Daly TE, Zimmerman S (1980) Radiation necrosis of the mandible: A 10 year study. I. Factors influencing the onset of necrosis. Int J Radiat Oncol Biol Physics 6: 543-548.

38 Granstromm G, Bergstrom K, Tjellstrom A, Branemark PI (1994) A detailed analysis of titanium implants lost in irradiated tissue. Int $J$ Oral Maxillofac Implants 9: 653-662.

39 Jacobsson MG, Jonsson AK, Albrektsson TO, Turesson IE (1985) Shortand long-term effects of irradiation on bone regeneration. Plast Reconstr Surg 76: 841-848. 
40 Ueda M, Kaneda T, Takahashi H (1993) Effect of hyperbaric oxygen therapy on osseointegration of titanium implants in irradiated bone: A preliminary report. Int J Oral Maxillofac Implants 8: 41-44.

41 Watzinger F, Ewers R, Henninger A, Sudasch G, Babka A, et al. (1996) Endosteal implants in the irradiated lower jaw. J Craniomaxillofac Surg 24: 237-244.

42 Watzinger F, Ewers R, Henninger A, Sudasch G, Babka A, et al. (1996) Endosteal implants in the irradiated lower jaw. J Craniomaxillofac Surg 24: 237-244.

43 Jisander S, Grenthe B, Alberius P (1997) Dental implant survival in the irradiated jaw: A preliminary report. Int J Oral Maxillofac Implants 12: 643-648.

44 Ali A, Patton DW, El-Sharkawi AM, Davies J (1997) Implant rehabilitation of irradiated jaws: A preliminary report. Int J Oral Maxillofac Implants 12: 523-526.

45 Ekert SE, Desjardins RP, Keller E, Tolman DA (1996) Endosseous implants in an irradiated tissue bed. J Prosthet Dent 76: 45-49.

46 Niimi A, Ueda M, Keller EE, Worthington P (1998) Experience with osseointegrated implants placed in irradiated tissues in Japan and in the United States. Int J Oral Maxillofac Implants 13: 407-411.

47 Andersson G, Andreasson L, Bjelkengren G (1998) Oral implant rehabilitation in irradiated patients without adjunctive hyperbaric oxygen. Int J Oral Maxillofac Implants 13: 647-654.

48 DePaola LG, Peterson DE, Overholser CD (1996) Dental care for patients receiving chemotherapy. J Am Dent Assoc 112: 198-203.

49 RuggieroSL, Mehrotra B, Rosenberg TJ, EngroffSL(2004)Osteonecrosis of the jaws associated with the use of bisphosphonates: A review of 63 cases. J Oral Maxillofac Surg 62: 527-534.

50 Marx RE (2003) Pamidronate (Aredia) and zolendronate (Zometa) induced avascular necrosis of the jaws: A growing epidemic. J Oral Maxillofac Surg 61: 1115-1117.

51 Bagan JV, Murillo J, Jimenez Y (2005) Avascular jaw osteonecrosis in association with cancer chemotherapy: Series of 10 cases. J Oral Pathol Med 34: 120-123.

52 Migliorati CA (2003) Bisphosphonates and oral cavity avascular bone necrosis. J Clin Oncol 21: 4253-4254.

53 Kapitola J, Zak J, Lacinova Z, Justova V (2000) Effect of growth hormone and pamidronate on bone blood flow, bone mineral and IGF-I levels in the rat. Physiol Res 49: S101-S106.

54 Kapitola J, Zak J (1998) Effect of pamidronate on bone blood flow in oophorectomized rats. Physiol Res 47: 237-240.

55 Fournier P, Boissier S, Filleur S (2002) Jisphosphonates inhibit angiogenesis in vitro and testosterone-stimulated vascular regrowth in the ventral prostate in castrated rats. Cancer Res 62: 6538-6544.

56 Santini D, Vincenzi B, Avvisati G (2002) Pamidronate induces modifications of circulating angiogenic factors in cancer patients. Clin Cancer Res 8: 1080-1084.

57 Starck WJ, Epker BN (1995) Failure of osseointegrated dental implants after diphosphonate therapy for osteoporosis: A case report. Int J Oral Maxillofac Implants 10: 74-78.

58 Kribbs PJ (1990) Comparison of mandibular bone in normal and osteoporotic women. J Prosthet Dent 63: 218-222.

59 BirkenfeldL, Yemini M, Kase NG, Birkenfeld A(1999) Menopauserelated oral alveolar bone resorption: A review of relatively unexplored consequences of estrogen deficiency. Menopause 6: 129-133.
60 Payne JB, Reinhardty RA, Nummikoski PV, Patil KD (1999) Longitudinal alveolar bone loss in postmenopausal osteoporotic/osteopenic women. Osteoporosis Int 10: 34-40.

61 Fujimoto T, Niimi A, Sawai T, Ueda M (1998) Effects of steroidinduced osteoporosis on osseointegration of titanium implants. Int J Oral Maxillofac Implants 13: 183-189.

62 Nasu M, Amano Y, Kurita A, Yosue T (1998) Osseointegration in implant-embedded mandible in rats fed calcium-deficient diet: A radiological study. Oral Dis 4: 84-89.

63 Blomqvist JE, Alberius P, Isaksson S, Linde A, Hansson BG (1996) Factors in implant integration failure after bone grafting: An osteometric and endocrinologic matched analysis. Int J Oral Maxillofac Surg 25: 63-68.

64 August M, Chung K, Chang Y, Glowacki J (2001) Influence of estrogen status on endosseous implant osseointegration. J Oral Maxillofac Surg 59: 1285-1289.

65 Cooper LF (2000) Systemic effects of alveolar bone mass and implications in dental therapy. Periodontol 2000 23: 103-109.

66 Beikler T, Flemming TF (2003) Implants in the medically compromised patient. Crit Rev Oral Biol Med 14: 305-316.

67 Nevins ML, Karimbux NY, Weber HP, Giannobile WV, Fiorellini JP (1998) Wound healing around endosseous implants in experimental diabetes. Int J Oral Maxillofac Implants 13: 620-629.

68 lyama S, Takeshita F, Ayukawa Y, Kido MA, Suetsugu T, et al. (1997) A study of the regional distribution of bone formed around hydroxylapatite implants in the tibiae of streptozotocin-induced dietetic rats using multiple fluorescent labeling and confocal laser scanning microscopy. J Periodontol 68: 1169-1175.

69 Smith RA, Berger R, Dodson TB (1992) Risk factors associated with dental implants in healthy and medically compromised patients. Int J Oral Maxillofac Implants 7: 367-372.

70 Kapur KK, Garrett NR, Hamada MO (1998) A randomized clinical trial comparing the efficacy of mandibular implant-supported overdentures and conventional dentures in diabetic patients. Part I: Methodology and clinical outcomes. J Prosthet Dent 79: 555-569.

71 Fiorellini JP, Chen PK, Nevins MI, Nevins ML (2000) A retrospective study of dental implants in diabetic patients. Int J Periodontics Restorative Dent 20: 366-373.

72 Peled M, Ardekian L, Tagger-Green N, Gutmacher Z, Matchtel EE (2003) Dental implants in patients with type 2 diabetes mellitus: A clinical study. Implant Dent 12: 116-122.

73 World Workshop in Periodontics 1996 (1996) Consensus Report: Implant therapy II. Ann Periodontol 1: 816-820.

74 Sbordone L, Barone A, Ramaglia L, Ciaglia RN, lacono VJ (1995) Antimicrobial susceptibility of periodontopathic bacteria associated with failing implants. J Periodontol 66: 69-74.

75 Dent CD, Olson JW, Farish SE (1997) The influence of preoperative antibiotics on success of endosseous implants up to and including stage II surgery: A study of 2,641 implants. J Oral Maxillofac Surg 55: 19-24.

76 Blanchaert RH (1998) Implants in the medically challanged patient. Dent Clin North Am 42: 35.

77 Morris HF, Ochi S, Winkler S (2000) Implant survival in patients with type 2 diabetes: Placement to 36 months. Ann Periodontol 5: 157-165.

78 Rosenlicht JL (1999) Indications and contraindications for sinus grafting. In: Jensen OT (ed.). The Sinus Bone Craft. Chicago: Quintessence, pp: 7-15. 